\title{
Microcomputers big and little: Selecting a low-cost laboratory computer network
}

\author{
EVAN L. BROWN and KENNETH DEFFENBACHER \\ University of Nebraska at Omaha, Omaha, Nebraska 68101
}

\begin{abstract}
Characteristics of microcomputers are described affecting suitability for low-cost laboratory network use. Assuming a network of small microcomputers with interfaces for applications and a larger one for program development, 6502-based hobbyist computers appear cheapest at this time, while microcomputers imitating minicomputers have the best hardware and software support.
\end{abstract}

For purposes of this article, a microcomputer is a computer that uses a microCPU, a central processing unit (CPU) that consists of an integrated circuit in a single chip. Although this definition will be abandoned as more powerful single-chip CPUs are developed for use in larger computers, the distinction is an important one, since at present microcomputers are slower and cheaper than computers using CPUs composed of several chips. Computers with single-chip CPUs may generally be cheaper than traditional minicomputers and mainframe computers, but they vary considerably in cost and capabilities. A minimal system can consist of a CPU, its clock and support chips, enough memory to run a short program in machine language, interfaces for input and output (I/O), and, perhaps, a keyboard, display unit, and tape recorder. Krausman (1976) describes such a system for laboratory use, together with difficulty of programming. A medium configuration adds enough memory to run programs in BASIC or assembly language and can include a cathode-ray tube (CRT) terminal. Systems for program development can have additional memory, a disk storage unit, a hard-copy terminal, and numerous interfaces. Prices range from a few hundred dollars for a minimal system to several thousand for a microcomputer suitable for program development.

As microcomputers become more familiar pieces of laboratory apparatus and as more applications are exploited, it seems likely that psychology laboratories will either have timesharing or several microcomputers. Timesharing, although sensible in minicomputers, is less attractive with micros, since it does not decrease the costs of peripherals and/or memory. In addition, timesharing is not possible with more than one application requiring fast real-time processing. Future psychology laboratories may well find a need for multiple microcomputers.

A version of this paper was presented at the Seventh National Conference on the Use of On-Line Computers in Psychology, Washington, D. C., November 1977. Reprint requests should be addressed to Evan Brown, Department of Psychology, University of Nebraska at Omaha, Omaha, Nebraska 68101.
This article addresses considerations involved in selecting networks of microcomputers for use in the laboratory. The article may be useful to persons selecting a single microcomputer, but there are additional considerations involved in selecting microcomputers to be most compatible in a network.

\section{CHARACTERISTICS OF MICROCOMPUTER CENTRAL PROCESSING UNITS}

\section{Software Characteristics}

The CPU determines the availability of programming languages and program packages. BASIC and assembler languages are available for many, but not all, microCPUs and interpreters, and compilers are beginning to appear for FORTRAN, FOCAL, and COBOL. Special languages exist for microcomputers with limited memory. In addition, CPUs differ in the ease with which they may be programmed and the speed with which they run programs. Ease of programming may be affected both by the instruction set of the CPU (the set of commands it understands) and the availability of program development aids, such as monitor and editor programs for use on the computer itself and cross-assemblers and emulator programs that allow program development using a larger computer with a different CPU. CPU speed matters for some applications, but not others. Most on-line applications in psychology are slow enough to run on any CPU; exceptions include the processing of electrophysiological evoked potentials and digital audio.

\section{Hardware Characteristics}

Hardware characteristics of microCPUs are addressed in some detail by Peatman (1977). Most microCPUs are designed to be compatible with TTL (transistortransistor logic) chips, but a few are CMOS (common metal oxide semiconductor) and designed to be used with other CMOS chips for support. Although TTL is fine for operation from line current, the CMOS varieties take much less electricity and are preferred for battery-powered applications. MicroCPUs vary in 
the number of additional chips required to do a job, and to interface memory and $\mathrm{I} / \mathrm{O}$ devices. In a small system, these support chips may represent a large proportion of the cost. While most microCPUs are quite inexpensive (about $\$ 20$ ), the price of a minimal system may vary substantially from CPU to CPU. With larger systems, memory represents a greater proportion of the cost. The microCPU may affect this cost in several ways. Some CPUs can be used readily with dynamic memory, memory that requires periodic recharging to hold information; others require more expensive, static memory. The cost of memory is also affected by the clock speed of the CPU, with memory chips that can respond rapidly enough for a 4-megaHz clock costing more than slower memory. Finally, although it is difficult to obtain much comparative information, CPUs differ in the amount of memory needed for specific applications, and this clearly affects cost (see Barden, 1976, p. 87, for comparisons on three programs).

\section{CHARACTERISTICS OF MICROCOMPUTER SYSTEMS}

\section{Software Characteristics and Memory}

The software capabilities of a microcomputer are so strongly influenced by the amount and type of memory storage that these two factors generally must be considered together. Rudimentary microcomputers, ones costing less than about $\$ 300$, can only be programmed in machine language because of limited memory, although some make this not too arduous by permitting entry in octal (good) or hexadecimal (better) form, rather than as a string of ones and zeros. To obtain a reduced form of BASIC or a simple assembler requires at least $2 \mathrm{k}$ bytes of solid-state, random access memory (RAM), and more likely $4 \mathrm{k}$ (about $\$ 150$ ), just to interpret or assemble the language. A relatively full form of BASIC and working space to use it requires $12-16 \mathrm{k}$ of RAM, costing about $\$ 500$. RAM memory, however, does not persist when the power is off and is too expensive to use for long-term storage of programs or data.

More permanent storage methods vary considerably from computer to computer and also influence programming. At present, floppy disk systems are the most convenient forms of permanent yet erasable memory in systems of reasonable price. A separate disk storage unit costing perhaps $\$ 1,000$ permits storage and rapid access to $300 \mathrm{k}$ bytes or more on a spinning, flexible magnetic disk. This is useful for program development, for running programs in stages that are too large to store in RAM at one time, and for storing large amounts of data or large numbers of programs. An adequate substitute for storing data or programs, although not usually the other uses, is a tape recorder. With interface, a tape recorder might cost $\$ 100$. Two methods for storing information more permanently should be mentioned. At present, microcomputers differ in the amount of permanent, nonerasable solidstate memory contained and the uses made of it. Some may have no read-only memory (ROM). Others may have enough to call up the keyboard, or to enter information from a disk or tape recorder; still others have BASIC or an assembler in ROM. The last type of microcomputers are the easiest to use short of a disk system, but they lack a disk's flexibility. ${ }^{1}$ Within the year, it is generally expected that magnetic bubble memory systems will become available, with storage capacities approaching a disk system. With bubble memories, access is much faster and programming is easier (Helmers, 1977).

Microcomputers also differ in software support provided by the manufacturer. Some manufacturers have developed extensive software; others provide little or no software, or software of low quality. While it is possible to buy a computer of one type and run software developed by a different manufacturer for a computer with the same CPU, this can be expensive. Software charges for users of a manufacturer's hardware vary. Where this is apt to matter most for laboratory applications is in the availability of good forms of BASIC and other languages and good assemblers, cross-assemblers, monitors, and editors. Unless one can afford one of the relatively expensive microcomputer versions of a minicomputer, packages of programs specifically for laboratory work are unlikely to be available.

\section{Hardware Characteristics Other than Memory}

Microcomputers differ in available hardware support as well as software. This variation is most obvious with available peripherals and interfaces, but available service support is at least as important. Unless a unit has a technician, service is likely to hinge on the vendor. So the choice of computer hardware may be restricted to nearby vendors with adequate repair facilities and knowledgeable personnel. An excellent microcomputer purchased inexpensively through the mail is unlikely to be a better buy if there is no one available to help set it up or repair it. Even with technical support, the mail option should be approached with caution. Some manufacturers are notorious for taking orders in advance of production. A local vendor may have most components in stock and know which firms can be relied upon to deliver the others.

Input/output ports and interfaces for standard keyboards, printers, and alphanumeric video displays are available for most microcomputers. Laboratory interfaces for connecting switch inputs and outputs to lights or operant dispensers are lacking. Psychologists who use logic modules with operant equipment may wish to use switch inputs and power drivers for interfacing and acquire TTL converters from the manufacturer of the modules. Coulbourn Instruments, 
for example, sells six-channel converters for both input and output, at about $\$ 100$ each. A CRC Engineering XPRES System set of microcomputer-driven relays (about \$200) can be acquired for output, the only such board that we have seen for micros in general. This system requires that users construct their own switch debouncer from available circuits (e.g., Lancaster, 1974, pp. $203 \mathrm{ff}$.). Otherwise, such capabilities are available primarily for some microcomputer versions of minicomputers.

Analog-to-digital converters and digital-to-analog converters are in better supply. But only a small number of manufacturers make them, so the choice of a microcomputer for physiological or auditory uses might well hinge on the availability of analog conversion. For visual applications, the availability of video graphics can be a deciding factor. For department-wide use, microcomputers are available with both analog and video capabilities, including some that combine the two to provide a computer-based storage oscilloscope. Availability of real-time clocks and vectored interrupts is spotty. Although vectored interrupts may be necessary only in timesharing, and a clock can be avoided by programming timing loops, both can be helpful in programming and in on-line operations.

\section{COMBINING MICROCOMPUTERS IN A NETWORK}

Microcomputers can be mixed and matched in various ways, but it is most economical to avoid duplication of expensive components, such as terminals, printers, and disk storage units. Often, the best way to do this is to imitate manufacturers that use microcomputers for process control (cf. Osborne, 1976, Chapter 20). For process control, a manufacturer may buy large numbers of small micros, together with one large micro using the same CPU. The large micro, with a terminal, printer, and disk storage, is then used to develop programs for the small micros, and the latter then do the work in process control. Similar procedures may be followed in designing products that include a microCPU and associated chips, such as some microwave ovens, cash registers, and automobiles. A microcomputer network for a psychology department can be set up economically in the same way, with a single large micro for program development and tasks requiring large amounts of memory, and low-cost microcomputers to service other applications.

Since cross-assemblers and emulation programs exist to permit program development for microcomputers using mainframes or minis, it might be supposed that a large micro would be unnecessary. For some applications this is true. However, the micro permits interfaces and peripherals to be used on-line during program development. As a result, problems caused by them or by their interactions with the computer can be dealt with as they arise. This is of particular importance in high.-speed signal processing applications, but it can be useful in any on-line situation. Still, program development that excludes interfaces is better than no program development at all, so a user may wish to use the emulator and cross-assembler when a larger micro is unavailable, or to supplement a larger micro.

\section{MICROCOMPUTER CENTRAL PROCESSING UNITS SUITABLE FOR LABORATORY NETWORKS}

Dozens of microCPUs are available (see Osborne, 1976, for detailed descriptions and comparisons). At present, however, only five or six seem suited for low-cost networks involving one large micro for program development and several smaller ones for on-line applications. Most of the other microCPUs fail the test at the program development level: They are not inexpensive in configurations suitable for program development. These include some relatively rudimentary CPUs designed for use in appliances, CPUs unsuited to large-scale data processing in BASIC; but they also include some general purpose CPUs that have not been used in suitable systems. The latter may become viable alternatives in the future. Among the alternatives, there are some capable CPUs whose only problem is cost; these include microCPUs that imitate the CPUs of minicomputers. We shall return to them momentarily.

\section{MicroCPUs for Low-Cost Networks}

CPUs best suited to low-cost networks are those most popular with computer hobbyists. Both researchers and hobbyists need a CPU that can be used with inexpensive simple configurations and in large-scale data processing using high-level languages such as BASIC. A hobbyist is likely to start with a simple configuration and expand to a program development system. Hobbyist requirements make available much more equipment at a much lower price than would ever occur on the basis of laboratory demands. Game and hobbyist needs also mean that analog converters are more likely to be designed for TV games than physiological applications, and relays designed for electric trains instead of Skinner boxes. But this is a minor problem. A researcher can generally find micros that meet his/her research specifications.

The oldest hobbyist's CPU in current use is the Intel 8080 ; it is also the most widely used microCPU and has the most extensive available software and hardware. Compared to others, the Intel 8080 has two disadvantages: Its instruction set is difficult to understand, a problem only in machine language or assembler use, and it requires more support chips (Osborne, 1976). The latter increases the price of a large system slightly, but has more serious effects in minimal systems, where an 8080-based computer may cost twice as much as the cheapest competition. If one does choose 8080-based computers, the MITS designed 
Altair bus system is shared by several companies using $8080 \mathrm{CPUs}$, and has the largest variety of special-purpose boards, such as analog converters. Having such a common bus is particularly important for combining components in a large program development system. It can also make it easier to transfer interfaces and peripherals from computer to computer within a network. Use of the Altair bus line arrangement increases the cost, in that more efficient methods of construction have been developed, particularly for minimal systems.

Because of limitations in the 8080 , several variants have been developed. The Zilog Z-80 has the reputation of being the fastest hobbyist CPU and there is evidence that it may be faster than the 8080 at BASIC (Rugg \& Feldman, 1977). It also requires fewer support chips than the 8080 (Osborne, 1976), although price advantages have not generally been apparent. The Z-80 also understands about twice as many instructions as the 8080 , including instructions that simplify signal analysis programming. The $\mathrm{Z} 80$ is able to run 8080 programs with minor modification, and converters exist to adapt any 8080-based computer to Z-80 use. The Intel 8085 is an 8080 variant that has only just appeared. Since the 8085 reduces the numbers of support chips necessary, while remaining program compatible with the 8080 , it may be particularly useful for minimal systems. Another 8080 variant is the Intel 8048 , which requires very few support chips. As its instructions are a subset of the 8080 's instructions, machine language programs for it may be developed using an 8080,8085 , or Z-80. Given the 8048 's small instruction set, however, programs should be simple.

The second oldest of current microCPUs, and second most widely used, is the Motorola 6800 . As with the 8080 , it is available in systems ranging from the minimal to those suitable for program development. Although it has less software support and less hardware available, the 6800 can be used to set up a network. Compared to the 8080 , the 6800 has a more readily understood instruction set and needs fewer support chips. It is superior to the Intel 8080 for minimal systems, particularly those using machine or assembly language. Unfortunately, its more readily understood instruction set is also less powerful than that of the 8080 , and it is slower to run BASIC programs (Rugg \& Feldman, 1977).

The MOS Technology 6502 is a variant of the Motorola 6800 . The MOS 6502 reduces the number of support chips required, allowing still lower prices in minimal systems (Osborne, 1976). Its instruction set is only slightly more difficult to understand and much more powerful (Osborne, 1976). The 6502 seems to rival the Z-80 as hobbyist speed champion (Barden, 1976; Rugg \& Feldman, 1977) and seems nearly as well suited to electrophysiological and auditory signal analysis. At present, the 6502 seems to offer the lowest priced possibility for a network, one that equals the $8080-, 8085$, and Z-80-based networks in capabilities, if not in hardware or software support. One 6502-based computer in the low-priced range is the KIM-1, which costs about $\$ 250$ at the present time, requires a power supply costing less than $\$ 50$, and has all one might need for running most simple machine language programs: a monitor in ROM, 1000 bytes of RAM working memory, a small keyboard and display, two interval timers, interfaces for terminals and tape recorders, and $15 \mathrm{I} / \mathrm{O}$ lines (for detailed evaluation, see Tripp, 1977). To run BASIC on KIM-1, it is necessary to add memory. Ohio Scientific Instruments (OSI) is one of several companies making a computer for use with BASIC in the $\$ 500$ range. OSI also seems (at the present time) to be the only 6502-based computer company to offer an analog converter, video graphics, and a disk storage system within the same product line and on a common bus system. Others can be expected to do so, however, in the near future.

At present one must choose CPUs for networks carefully, because most manufacturers make computers available with but one choice of CPU. This may change to allow more freedom in the future. Digital Group and Microcomputer Associates (Jolt) allow one to choose among hobbyist CPUs by substituting CPU boards. OSI allows a similar choice under either hardware or program control, using a single board; OSI also provides a more expensive option (about $\$ 1,000$ ), permitting simultaneous multiprocessor use of a Z-80, 6502, 6800, and 6100 (a PDP-8 equivalent, to be discussed below). Using one of these for program development, the researcher can develop programs for other computers in a system mixing CPUs.

\section{Some More Expensive Alternatives}

As noted, there are several other microcPUs in addition to those of hobbyist interest that are available in minimal configurations and in a form suitable for program development, although systems based on them tend to be more expensive. These include CPUs that take the instruction sets of minicomputers, an appealing feature for minicomputer owners. The greater availability of software and peripherals for minicomputers can make micros imitating them useful despite the cost. MicroCPUs that take minicomputer instruction sets include the Intersil 6100 (PDP-8), the Data General MicroNova and Fairchild 9440 (Nova) and the Digital Equipment Corporation (DEC) LSI-11 (PDP-11). (Corresponding minicomputers are given in parentheses.) While computers based on these CPUs tend to be expensive even in minimal configuration, PDP-8 users and others interested in PDP-8 software might note exceptions involving the 6100 . Although it needs several extra-cost options for most uses, the $\$ 300$ Intersil Intercept Jr. provides a simple keyboard and digital display, a small amount of memory, and the 
opportunity for a small amount of expansion (see Stark, 1977, for detailed evaluation). Since the Intercept Jr. is one of the few CMOS microcomputers available, it should also be considered by those needing long-term battery operation. PDP-8 users might consider the fact that the 6100 may be used with other computers, such as the multiprocessor OSI. PDP-11 owners might consider the LSI-11-based Heathkit H-11. The H-11 costs considerably more than hobbyist micros with similar features, and is only available as a kit; DEC supplies some of the peripherals and laboratory interfaces. However, it appears to be faster than the hobbyist micros (Rugg \& Feldman, 1977) and has DEC's software support.

\section{CONCLUSION}

Several alternatives exist at this time for a low-cost laboratory computer network. Computers designed for hobbyists provide lowest costs, and this seems likely to be the situation in the future, given the size of the hobbyist market. Hobbyist computers based on the 8080 and its variants have the most hardware and software available; computers based on the 6502 seem to be cheaper for network use and have adequate support for most laboratory applications. These comparisons, however, are based on current information, and the microcomputer field is changing and expanding rapidly.

\section{REFERENCES}

BARDEN. W., JR. How to buy and use minicomputers and microcomputers. Indianapolis: Sams, 1976.

HeLmers. C. This elephant never forgets. Byte, 1977, 2 (7) 6. $58-61$

Krausman, D. T. The microcomputer kit: An excellent small system development tool. Behavior Research Methods \& Instrumentation, 1976, 8, 501-507.

LANCASTER, D. TTL cookbook. Indianapolis: Sams, 1974.

OSBORNe. A. An introduction to microcomputers, Volume 11: Some real products. Berkeley: Osborne, 1976.

Peatman, J. B. Microcomputer-based design. New York: McGraw-Hill, 1977.

Rugg, T., \& Feldman, P. BASIC timing comparisons. . .revised and updated. Kilobaud, 1977, 1 (10), 20.25.

STARK, P. A. Prototyping systems exposed. . . a revealing look at the Intercept Jr. Kilobaud, 1977, 1 (5), 66-77.

TRIPP, R. M. Is the Kim-1 for every-1? Kilobaud, 1977, 1 (8), 56.59

\section{NOTE}

1. Although slower and less flexible than a disk system for program development and storage, a microcomputer with BASIC in ROM, 4k of RAM working memory, plus a tape recorder, a CRT, and their interfaces can be had for about $\$ 600-\$ 750$. We have in mind the 6502-based Commodore PET, the OSI IIP, and the Z-80-based Radio Shack TRS-80. While the OSI is the most expensive of the three, it is the only one with an analog converter at this time. It also has a standard keyboard (the PET does not), 8k BASIC (the TRS-80 has $4 \mathrm{k}$ BASIC), and is the only one to share a common bus with a larger program development system. 